de Gràcia i Pérez, Enric.

Alumno, Universitat Oberta de Catalunya, estudiante en el máster universitario de Humanidades: arte, literatura y cultura contemporánea.

\title{
colabocrea.net como experiencia en el arte de participación y las redes sociales.
}

\author{
TIPO DE TRABAJO \\ Comunicación. \\ PALABRAS CLAVE \\ Redes sociales, arte relacional, participación.
}

KEY WORDS

Social networks, relational art, participation.

\section{RESUMEN}

Análisis sobre el proyecto Col/labo (colabocrea.net) en el entorno del arte de participación y las redes sociales (virtuales). Realizado desde la experiencia directa adquirida durante el proceso de creación y tras un periodo de inactividad (de este) posterior. Las redes sociales virtuales, como las redes sociales en la sociología, suponen un tema de estudio de actualidad sobre el que vamos adquiriendo nuevas perspectivas de forma continua. Col/labo, como plataforma para desarrollar proyectos artísticos de forma colaborativa y/o participativa, recoge, como no puede ser de otra manera, diversas experiencias de participación. Pero no todas ellas son directas, pues también las hay indirectas. Es decir, personas que interactúan con el proyecto (o con otros proyectos, no es una característica exclusiva de este proyecto), sin tener constancia de ello, y por lo tanto, sin la intención de participar, tan solo por curiosidad, casual o no, o buscando el disfrute.

\section{ABSTRACT}

Analysis about the project Col/labo (colabocrea.net), in the environment of participation art and social networks (virtual ones). Accomplished from the direct experience acquired during the creation process and also after an inactivity period after (of this). The virtual social networks, similarly to social networks in sociology, suppose a current topic of study, on which we are acquiring new points of views continuously. Col/labo, as a platform to develop artistic projects as a collaborative and/or participative way, gather, at the same time, various experiences of participation. But not all of them are direct, but also indirect ones. People who interact with the project (or with other projects, that's not an exclusive property of this project), without knowing about it, and therefore, who do not intend to participate, just for curiosity, casual or not, or for their own pleasure.

\section{INTRODUCCIÓN}

La idea de crear de forma colectiva parece contemporánea, pero no lo es. Lo cierto es que, en el arte, el acto de crear implica siempre a otras personas. El artista o la artista necesita de la alteridad, por lo que convoca a "otros" para que contemplen su obra, pues esta no será arte si no es compartida. Entonces, ¿’por qué y para quién creamos?

La idea de si la mirada, la presencia o la interacción del público forman parte de la obra o no, es un debate que (en el ámbito teórico), finaliza Bourriaud con su definición de estética relacional, cuando afirma que es una "teoría estética que consiste en juzgar las obras 
de arte en función de las relaciones humanas que figuran, producen o suscitan" ${ }^{1}$. A mediados del siglo XX, las performances, los happenings, el teatro y la danza nos mostraron, como diría Martín Prada, prácticas de "experiencias colectivizadas" ${ }^{2}$. Este (ya difunto) debate trata de deconstruir la idea tradicional del binomio autor/a-público, hacia una idea de co-autor/a, en la que se reconoce el papel importante del público. Pero hay un planteamiento aun más importante: el papel que tiene el arte en la sociedad. Aceptar el fin de este debate en favor del público implica un debilitamiento del modelo de espectáculo global, y en algunos casos especulativo, pues si crece la tendencia de participación, tenderá a bajar la de sobrenombres. Además de esta tendencia ascendente hacia la participación, hay también en esta época un acercamiento de Occidente a la cultura de lo colectivo (democracia, movimientos asamblearios, teorías de lo común, etc.), permitiendo así (en algunos casos) el reconocimiento de todas esas personas que aportan algo a la obra (como antiguamente era el caso de los obreros, discípulos y ayudantes). Esa tendencia ascendente, que evidentemente no ocupa la totalidad de las prácticas artísticas, se vuelve a dar en la actualidad, con un número cada vez mayor de trabajos publicados, la diferencia es que ahora, la tecnología nos permite saber con más exactitud que trabajos se están desarrollando, además de permitirnos participar en algunos de ellos.

\section{COL·LABO COMO EXPERIENCIA. ARTE DE PARTICIPACIÓN Y REDES SOCIALES}

Col $/$ labo ${ }^{3}$ es un proyecto que actúa como plataforma para desarrollar trabajos creativos, de forma colaborativa. Nace a partir de diferentes proyectos audiovisuales basados en el modelo de las cooperativas y del estudio de otros referentes artísticos contemporáneos. Además, se nutre de las teorías "Open Source" (Código abierto), como la Cultura Libre, las "Wikis" o las licencias "Creative Commons".

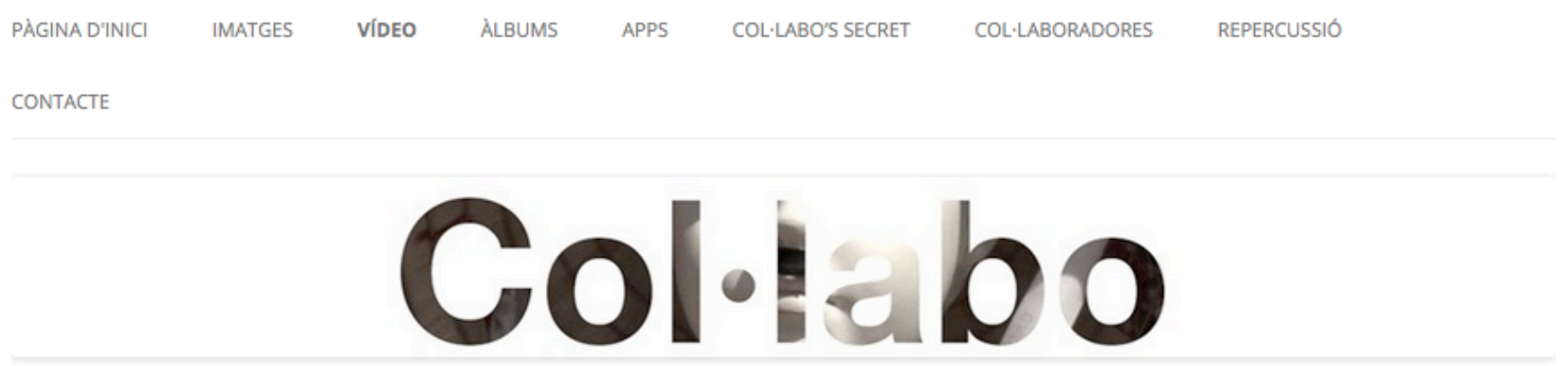

Captura de la página web del proyecto, colabocrea.net

La estructura del proyecto está sustentada en dos tipos de interacciones: el encuentro real y el virtual (ambos actúan de forma sinérgica). Los trabajos se desarrollan desde la práctica tradicional, que podemos identificar como presencial (física o real), pero también se usa otro tipo de herramientas como Twitter, Skype, o apps de dispositivos móviles para crear en un entorno de comunicación virtual (online). En el encuentro físico, la participación (entendida como la interacción voluntaria de los distintos individuos), se realiza de forma presencial. A partir del diálogo y la escucha, o bajo la forma de una un taller o una asamblea, se obtiene como fruto una idea (o ideas). El desarrollo de esta idea se continuará en distintas sesiones hasta conseguir su materialización. Durante este proceso de desarrollo, está abierta la incorporación de nuevas personas. Mi experiencia en este proyecto me ha permitido, dentro de unos límites, analizar algunos de los roles dentro del ámbito de la participación.

En primer lugar, encontramos a las personas que, mediante las redes sociales ${ }^{4}$ o el correo electrónico ${ }^{5}$, se ponen en contacto con el proyecto. Estas personas demandan participar en alguna de las propuestas que se están desarrollando en ese momento, u ofrecen nuevas ideas. Se trata de un modo de participación directa y horizontal basado la idea de Gobierno abierto ${ }^{6}$. Pese a que el contacto se realiza mediante herramientas virtuales, la práctica artística se puede realizar de forma presencial (se trata de la forma más tradicional) u online. Estas se realizan gracias a las llamadas "tecnologías de conectividad", que permiten el acceso a la participación

${ }^{1}$ Bourriaud, N. Estética Relacional (2008).

2 Juan Martín Prada utiliza el término de "momentos de experiencia colectivizada" en Otro tiempo para el arte. Página 50.

${ }^{3} \mathrm{Col} \cdot \mathrm{labo}$ es un proyecto presentado como parte de mi Trabajo fin de grado Art de participació $i$ cultura audiovisual. Un cas d'estudi: colabocrea.net (2014)

${ }^{4}$ Las redes sociales son una serie de herramientas online basadas en la web 2.0 que permiten la interconexión entre una multitud de usuarios. Reciben este nombre por su similitud al concepto sociológico, que conceptualiza lazos sociales reales.

${ }^{5}$ Todas las redes sociales y las formas de contacto se muestran en la web colabocrea.net

${ }^{6}$ Gobierno abierto es un libro coordinado por César Calderón y Sebastián Lorenzo, que ofrece un modelo de democracia más participativa con garantías de participación ciudadana. 
sin la necesidad de estar presente. De esta manera, todos los usuarios de Internet (es decir, todos aquellos y aquellas que no sufren la brecha digital) pueden, además de contactar con el proyecto y sugerir propuestas, cantar, tocar algún instrumento y enviar archivos o vídeo por streaming.

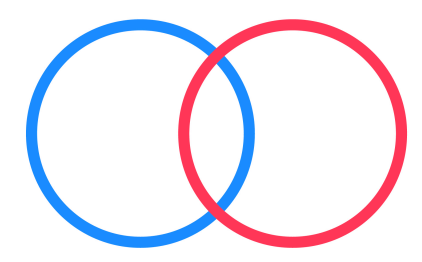

\section{Dibujo del lazo entre Col·labo (azul) y el/la participante (rojo)}

Estas personas crean una red, en la que otras personas conectadas a las primeras pueden observar los resultados publicados, y en algunos casos, interesarse por como participar. Por una parte, la red estaría motivada por intereses, pero aquello que llamamos redes sociales generan otro tipo de referentes, de los cuales no se tiene un perfil determinado. Esto es lo que hoy se llama influencers, personas que son referentes para otras, por identificación o aceptación. Se genera mediante la red una suerte de contactos físicos y contactos virtuales.

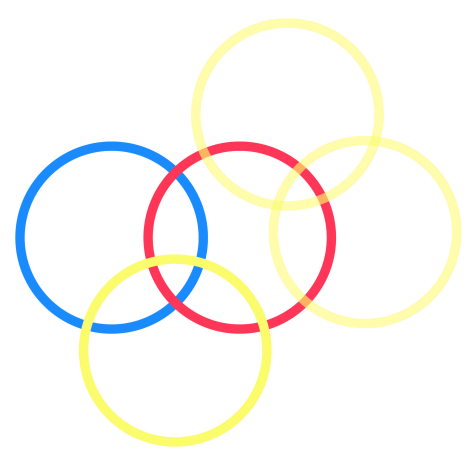

Dibujo del lazo entre Col·labo (azul), el/la participante (rojo) y contactos interesados (amarillo)

También encontramos personas que participan por "contacto". Me refiero a las personas cercanas al proyecto, o cercanas a otras personas que han participado en él. Hay que tener en cuenta que, inmediatamente después de que una persona participe, las personas cercanas a esta con un alto interés en crear, pasan a ser (en algunos casos y mediante la recomendación o la causalidad), colaboradores potenciales. En este caso, la red de contactos también se genera de la misma manera que en el ejercicio anterior, pero al tener un carácter más local (pues se trata de personas cercanas al proyecto o personas cercanas a estas personas), crece aun más tendencia hacia una red de contactos físicos (aunque ello no perjudica al número de contactos virtuales).

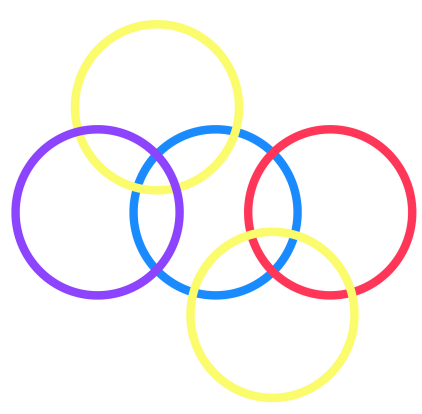

Dibujo del lazo entre Col·labo (azul), personas cercanas a este (morado), el/la participante (rojo) y contactos interesados (amarillo) 
De acuerdo con la introducción que he realizado al principio, acuso que en estos ejemplos se descartan todas las formas posibles de público, y que por tanto se deja fuera este otro modo de participación. Cada vez que se publica un trabajo, se activan las redes creadas. Aquí surge la parte más importante de las redes sociales de hoy. Si el trabajo se publica en Internet, puede ser visualizado (y reproducido) por miles de personas. Admito que este hecho es una obviedad, pero no por ello es menos importante, pues en el caso de las prácticas participativas y colaborativas abre una multitud de experiencias antes imposibles. Por ejemplo, en el caso de Col/labo, cuando se realiza una canción se publica en una página web llamada Bandcamp. Toda la información de ese trabajo se comparte en un perfil creado para el proyecto, y la página proporciona un enlace para compartirlo en las otras redes sociales. Además, el uso de etiquetas ayuda a que terceros encuentren las obras. Todo esto se reproduce de la misma manera con otra página llamada Soundcloud. Con una excepción importante, que no se comparte el enlace desde el proyecto. Siempre se usa Bandcamp con la finalidad de centralizar a los visitantes en un mismo sitio y no causar demasiadas interferencias. Desde el mes de julio, el proyecto ha estado prácticamente inactivo, pero el número de reproducciones y descargas en Soundcloud, que era casi nulo, no ha cesado.

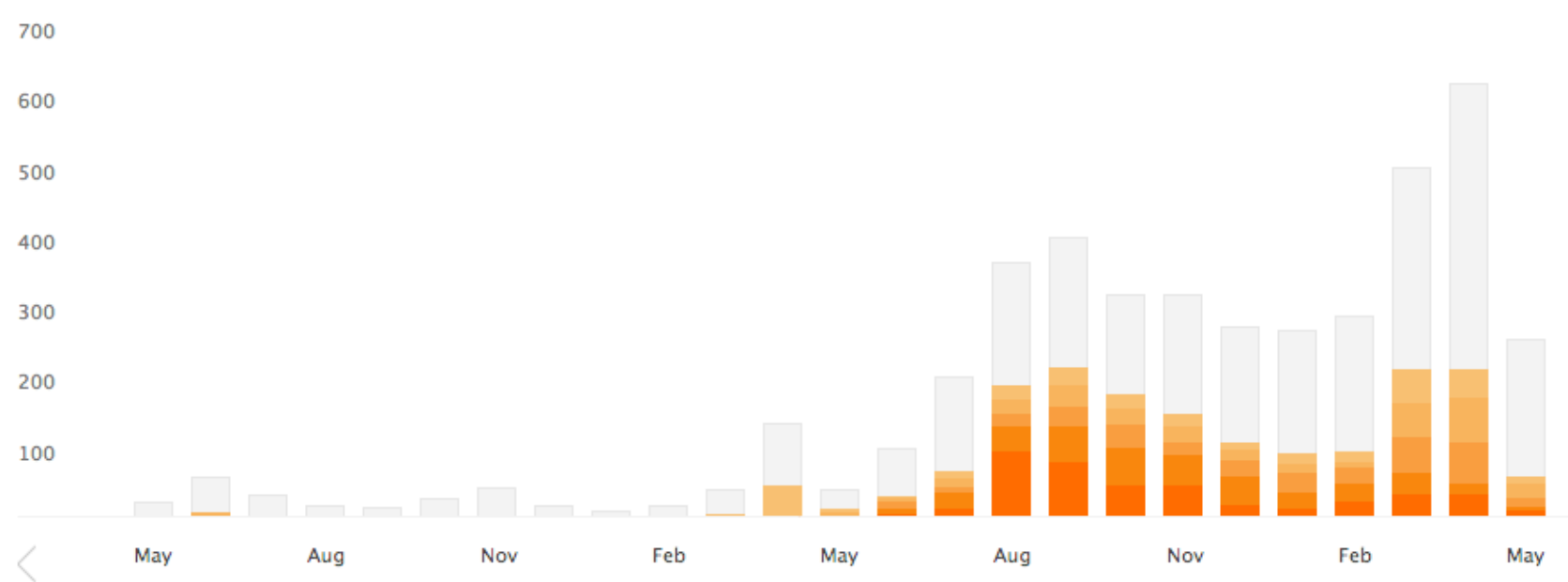

Estadísticas de Soundcloud. https://soundcloud.com/col-labo/stats/plays/2013-05-29/2015-05-10

Esto se debe a que uno de los proyectos, llamado Projecte Rizoma (Proyecto Rizoma, por hacer referencia a Deleuze y Guattari ${ }^{7}$ ), era realizar un álbum de música digital en el que, quien quisiera, podía elegir una canción de las publicadas anteriormente por Col/labo, realizar una portada, y yo me comprometía a publicar la música de esa canción sin letra, para que otras personas pudiesen escucharla, descargarla y/o utilizarla con otros fines diferentes a los del trabajo inicial (con letra). En este ejemplo, cada una de esas descargas, reproducciones, enlaces usados, etc. son parte del proyecto, pues se trataba de eso: generar un rizoma de usuarios interconectados que pudiesen participar, escuchar y ver lo que hacían los otros.

Varias canciones han llegado al tope de descargas (100), y el total de reproducciones en menos de un año supera las 4.000 . No son grandes cifras, pero no serían posibles si la red no funcionara como tal. Si buscas colabocrea.net en la red, aparece una gran cantidad de referencias que no tienen nada que ver con el proyecto. Hay muchas personas que se dedican a recoger información y publicarla en páginas temáticas o en blogs. Evidentemente hay aun una barrera tecnológica (y la no menos importante Ley de protección de datos, LOPD).

\section{¿TENDENCIA O PRÁCTICA DE MODA?}

Como vengo diciendo, en la actualidad hay todo tipo de practicas relacionales. No solo en el Estado español, también en el resto del mundo. Cada vez, la tendencia crece en favor de lo común. Vemos como las cooperativas, las prácticas hitchhiking online como Blablacar o Uber, el couchsurfing y el crowdfunding se convierten en alternativas (reales y horizontales) al modelo económico-social verticalmente rígido que aun persiste. Pero esto no quiere decir que se alimente de un fenómeno temporal pasajero. Seguro que existen prácticas dentro de esta corriente, con una finalidad no participativa, como lo son algunos espacios dentro de los medios de comunicación, que ofrecen tipos de participación para atraer a la audiencia (como son los espacios que muestran lo que comentan los usuarios de Twitter que utilicen determinada etiqueta (o Hastag).

\footnotetext{
${ }^{7}$ Deleuze y Guattari tienen un ensayo sobre el concepto "rizoma".
} 


\section{CONCLUSIÓN}

El arte, como lo ha hecho siempre, debe permanecer a su tiempo. Este, en concreto, se nutre de Internet, de las TIC, de la Cultura del Remix, y de la web 2.0. Todos estos factores tecnológico-sociales han posibilitado una sociedad que necesita ya estas prácticas de lo común, pues sustituyen a otras prácticas que por diversos factores (como lo ha sido la Crisis económica de finales de la primera década de los años 2000 hasta la actualidad), han dejado de ser funcionales. En la economía vemos los ejemplos del apartado anterior, en la política nos encontramos con lo que está pasando en el Estado español y en otros tantos países, donde aparecen nuevos partidos que fomentan el uso de asambleas y las plataformas de participación. En lo cultural, hay cada vez más artistas, realizadores y músicos que siguen este camino, como por ejemplo los documentales de la contra-información, o los que se realizan mediante plataformas como Verkami. También vemos como en lo social hay una multitud cada vez más interconectada, que exige un progreso hacia la igualdad y que exige y opina, en los espacios físicos, pero también en las redes sociales.

Esta realidad no se entiende impacto sin las tecnologías de la conectividad, que nos permiten estar conectados y expuestos a Internet de forma permanente, por lo que creo que en el arte de participación, o relacional, juegan un papel muy importante, abriendo una puerta a un espacio virtual amplio e interconectado con los otros usuarios que cruzan dicha puerta.

\section{FUENTES REFERENCIALES.}

BOURRIAUD, Nicolas. Estética relacional. Buenos Aires: Adriana Hidalgo editora, 2008.

CALDERÓN, Cesar, LORENZO, Sebastián (Coords.). Open Government. Gobierno abierto. Jaén: Algón editores, 2010.

DELEUZE, Gilles, GUATTARI, Félix. Rizoma. Valencia: Pre-textos, 1977.

DE GRÀCIA, Enric. Art de participació i cultura audiovisual. Un cas d'estudi: colabocrea.net [en línea]. Valencia: UPV, 2014. Disponible en Web: http://hdl.handle.net/10251/45299 [Consulta: 20 de abril de 2015].

PRADA, Juan Martín. Otro tiempo para el arte. España: Sendema, 2012.

PRADA, Juan Martín. Prácticas artísticas e Internet en la época de las redes sociales. España: Akal, 2012. 